\title{
SURFACE DISTRIBUTION OF M STARS WITH DIFFERENT IRAS COLOUR
}

\author{
Keiichi ISHIDA \\ Tokyo Astronomical Observatory, University of Tokyo \\ Mitaka, Tokyo 181, Japan \\ and \\ Mazlan OTHMAN \\ Physics Department, National University of Malaysia \\ Bangi, Malaysia
}

The surface distribution of $M$ stars is studied by differentiating them according to whether they show a circumstellar dust shell (CS) or not. Analysis shows that galactic latitudinal and longitudinal distributions are not determined by spectral subclasses alone. The study also indicates that the $M$ type stars with $C S$ have higher intrinsic luminosities in the $k$ band than those without CS. The M stars used in the study are obtained from the Two Micron Sky Survey catalogue (IRC) which is an unbiased sample with respect to the interstellar extinction. The CS feature is identified by the ratio of flux densities at 12 and $25 \mu \mathrm{m}$ in the IRAS point source catalog.

Table 1. Spectral composition of stars without and with CS.

\begin{tabular}{lrrr} 
& \multicolumn{2}{c}{$\begin{array}{c}\text { number of stars } \\
\text { without CS }\end{array}$ with CS } & total \\
\hline K and earlier & 1318 & 36 & 1354 \\
early M (M5 \& earlier) & 1650 & 614 & 2264 \\
late M (M6 \& later) & 325 & 1117 & 1442 \\
S type stars & 31 & 40 & 71 \\
carbon stars & 130 & 58 & 188 \\
total IRC stars identified & & & \\
with an IRAS point source & 3454 & 1865 & 5319 \\
& & &
\end{tabular}

